\title{
Estimating Long-Term Survival Outcomes for Tumor-Agnostic Therapies: Larotrectinib Case Study
}

\author{
Andrew Briggs ${ }^{a}$ Noman Paracha ${ }^{b}$ Katherine Rosettie ${ }^{c}$ Alexander Upton ${ }^{d}$ \\ Carsten Bokemeyer $^{\mathrm{e}}$ Ulrik Lassen $^{\mathrm{f}}$ Sean D. Sullivan ${ }^{9}$ \\ aLondon School of Hygiene and Tropical Medicine, London, UK; ${ }^{b}$ Bayer Pharmaceuticals, Basel, Switzerland;

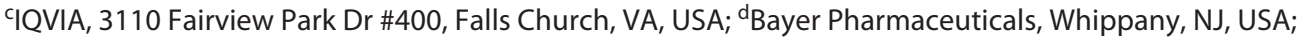 \\ eUniversity Hospital Hamburg-Eppendorf, Hamburg, Germany; fDepartment of Oncology, Rigshospitalet, \\ Copenhagen, Denmark; ${ }^{9}$ The CHOICE Institute, University of Washington School of Pharmacy, Seattle, WA, USA
}

\section{Keywords}

Tumor-agnostic therapies · Survival analysis · Long-term survival · Precision oncology

\begin{abstract}
Background: Larotrectinib is a precision oncology treatment for solid tumors with neurotrophic tyrosine receptor kinase (NTRK) gene fusions. Larotrectinib efficacy has been evaluated in single-arm basket trials with limited follow-up and sample sizes at the initial regulatory approval due to the rarity of solid tumors with NTRK gene fusion. Objectives: We aim to demonstrate that trends in progressionfree survival (PFS) and overall survival (OS) in survival data with longer follow-up may be predicted from long-term survival estimates from survival data with shorter followup, including predictions for median survival when it is not observed in the trial. Methods: Patient-level data were pooled from 3 clinical trials (NCT02122913, NCT02576431, and NCT02637687) using the 2018 and 2020 data cuts for the same subset of pediatric and adult patients. The Weibull distribution was selected for survival models. Survival predictions using 2018 data were compared to 2020 KaplanMeier (KM) curves. Results: A total of 102 patients repre-
\end{abstract}

karger@karger.com www.karger.com/ocl

Karger $\frac{1}{\%}$
(C) 2021 The Author(s)

Published by S. Karger AG, Basel

This is an Open Access article licensed under the Creative Commons Attribution-NonCommercial-4.0 International License (CC BY-NC) (http://www.karger.com/Services/OpenAccessLicense), applicable to the online version of the article only. Usage and distribution for commercial purposes requires written permission. senting 15 tumor types were included in the analysis, with a mean age of 37 years. When comparing PFS from the 2018 survival prediction to observed $2020 \mathrm{KM}$ data, the 12 -month PFS rate was identical (66.6\%). The 36-month PFS rate was lower for the 2018 prediction (35.3\%) compared to $2020 \mathrm{KM}$ data (44.4\%). The median OS had not yet been reached in either data cut but was predicted to be 90 months using the 2018 data. When comparing OS from the 2018 survival prediction to the observed $2020 \mathrm{KM}$ data, the 12 -month OS rate was $89.0 \%$ and $86.6 \%$ and the 48 -month OS rate was $67.2 \%$ and $63.0 \%$, respectively. Conclusion: Long-term PFS predictions deviated from observed PFS rates due to response differences across tumor types and heavy censoring towards the end of the survival curve. However, for OS, the 48-month survival prediction was consistent with the observed $2020 \mathrm{KM}$ estimate.

(c) 2021 The Author(s)

Published by S. Karger AG, Basel

\section{Introduction}

Tumor-agnostic therapies target specific genomic alterations when treating cancer, as opposed to the traditional paradigm of targeting the tumor site regardless of 
oncogenic driver. Tumor-agnostic therapies are typically investigated using single-arm basket trials due to the rarity of the genomic alterations they target [1].

Larotrectinib is a tumor-agnostic therapy and highly selective Tyrosine Receptor Kinase inhibitor that is approved in many countries world-wide including the USA and European Union for solid tumors with neurotrophic tyrosine receptor kinase (NTRK) gene fusions $[2,3]$. Due to the rarity of solid tumors with NTRK gene fusion, larotrectinib efficacy has been evaluated in single-arm basket trials with the initial regulatory approval and $\mathrm{Na}$ tional Institute for Health and Care Excellence health technology assessment based on the 2018 data cut with limited follow-up for overall survival (OS) and limited sample sizes [4-6], yet larotrectinib trials continue enrolling and following patients over time.

Assessment of long-term clinical effectiveness is necessary to inform clinical decision-making and involves the extrapolation of efficacy beyond the observed study period. Survival predictions are also valuable in estimating clinical benchmarks, such as median survival, when follow-up and the number of events are limited. The objective of the analysis was to investigate the consistency of long-term survival predictions for OS and progressionfree survival (PFS) for tumor-agnostic therapies, using larotrectinib as an example, with observed survival outcomes in a later data cut.

\section{Materials and Methods}

We used pooled adult and pediatric patient-level data from 3 larotrectinib clinical trials (Adult Phase I [NCT02122913], SCOUT [NCT02637687], and NAVIGATE [NCT02576431]) $(N=102)$ using the population included in the European label data cut, assessed by both the independent review committee and investigator [4-6]. The same subset of patients was analyzed using the 2020 data cut. Primary and secondary endpoints in the 3 larotrectinib trials include overall response rate, PFS, and OS [4-6].

Survival modeling is an established statistical method for estimating long-term outcomes from clinical oncology studies where the main outcome is the time to an event of interest [7]. The Weibull distribution was selected based on statistical fit (i.e., testing how alternative distributions best fit the observed data); this was the base case distribution for the larotrectinib National Institute for Health and Care Excellence health technology assessment submission [8].

Survival predictions using the 2018 data were compared to the 2020 Kaplan-Meier (KM) curves. Our basis for comparison of survival predictions to the $2020 \mathrm{KM}$ data was the time point at which $10 \%$ of patients were at risk of an event as the survival curves are reliable up to that time point [9]. Using this $10 \%$ cutoff, comparisons were made at 3 and 4 years for PFS and OS, respectively.

Long-Term Survival for Tumor Agnostic Therapies
Table 1. Patient characteristics common to both data cuts (2018 and 2020)

\begin{tabular}{|c|c|}
\hline Characteristic & Value $(N=102)$ \\
\hline \multicolumn{2}{|l|}{ Age } \\
\hline Mean (SD) & $37.1(26.6)$ \\
\hline \multicolumn{2}{|l|}{ Age category, $n(\%)$} \\
\hline$<1$ year & $9(8.8)$ \\
\hline$\geq 1$ and $\leq 5$ & $10(9.8)$ \\
\hline$>5$ and $\leq 11$ & $8(7.8)$ \\
\hline$>11$ and $\leq 17$ & $7(6.9)$ \\
\hline$>17$ and $\leq 44$ & $22(21.6)$ \\
\hline$>45$ and $\leq 64$ & $26(25.5)$ \\
\hline$>64$ and $\leq 74$ & $14(13.7)$ \\
\hline$>74$ & $6(5.9)$ \\
\hline \multicolumn{2}{|l|}{ Sex, $n(\%)$} \\
\hline Male & $54(52.9)$ \\
\hline Female & $48(47.1)$ \\
\hline \multicolumn{2}{|l|}{ Race, $n(\%)$} \\
\hline White & $74(72.6)$ \\
\hline Black & $5(4.9)$ \\
\hline Asian & $4(3.9)$ \\
\hline All others & 19 (18.6) \\
\hline \multicolumn{2}{|l|}{ Primary tumor site, $n(\%)$} \\
\hline Appendix & $1(1.0)$ \\
\hline Bone sarcoma & $2(2.0)$ \\
\hline Breast & $1(1.0)$ \\
\hline Cholangiocarcinoma & $2(1.0)$ \\
\hline Colon & $6(5.9)$ \\
\hline Congenital mesoblastic nephroma & $1(1.0)$ \\
\hline GIST & $4(3.9)$ \\
\hline IFS & $13(12.8)$ \\
\hline Lung & $7(6.9)$ \\
\hline Melanoma & $7(6.9)$ \\
\hline Pancreas & $1(1.0)$ \\
\hline Primary CNS & $9(8.8)$ \\
\hline Salivary gland & $17(16.7)$ \\
\hline Soft tissue sarcoma & $21(20.6)$ \\
\hline Thyroid & $10(9.8)$ \\
\hline \multicolumn{2}{|l|}{ Baseline ECOG, $n(\%)$} \\
\hline 0 & $47(46.1)$ \\
\hline 1 & $44(43.1)$ \\
\hline 2 & $11(10.8)$ \\
\hline \multicolumn{2}{|l|}{ Disease extent at enrollment, $n(\%)$} \\
\hline Locally advanced & $16(15.7)$ \\
\hline Metastatic & $77(75.5)$ \\
\hline Other & $9(8.8)$ \\
\hline \multicolumn{2}{|l|}{ Tumor stage at diagnosis, $n(\%)$} \\
\hline 1 & $10(9.8)$ \\
\hline ॥ & $16(15.7)$ \\
\hline III & $25(24.5)$ \\
\hline IV & $25(24.5)$ \\
\hline Not reported & $26(25.5)$ \\
\hline
\end{tabular}

SD, standard deviation; GIST, gastrointestinal stromal tumor; IFS, infantile fibrosarcoma; CNS, central nervous system; ECOG, Eastern Cooperative Oncology Group. 


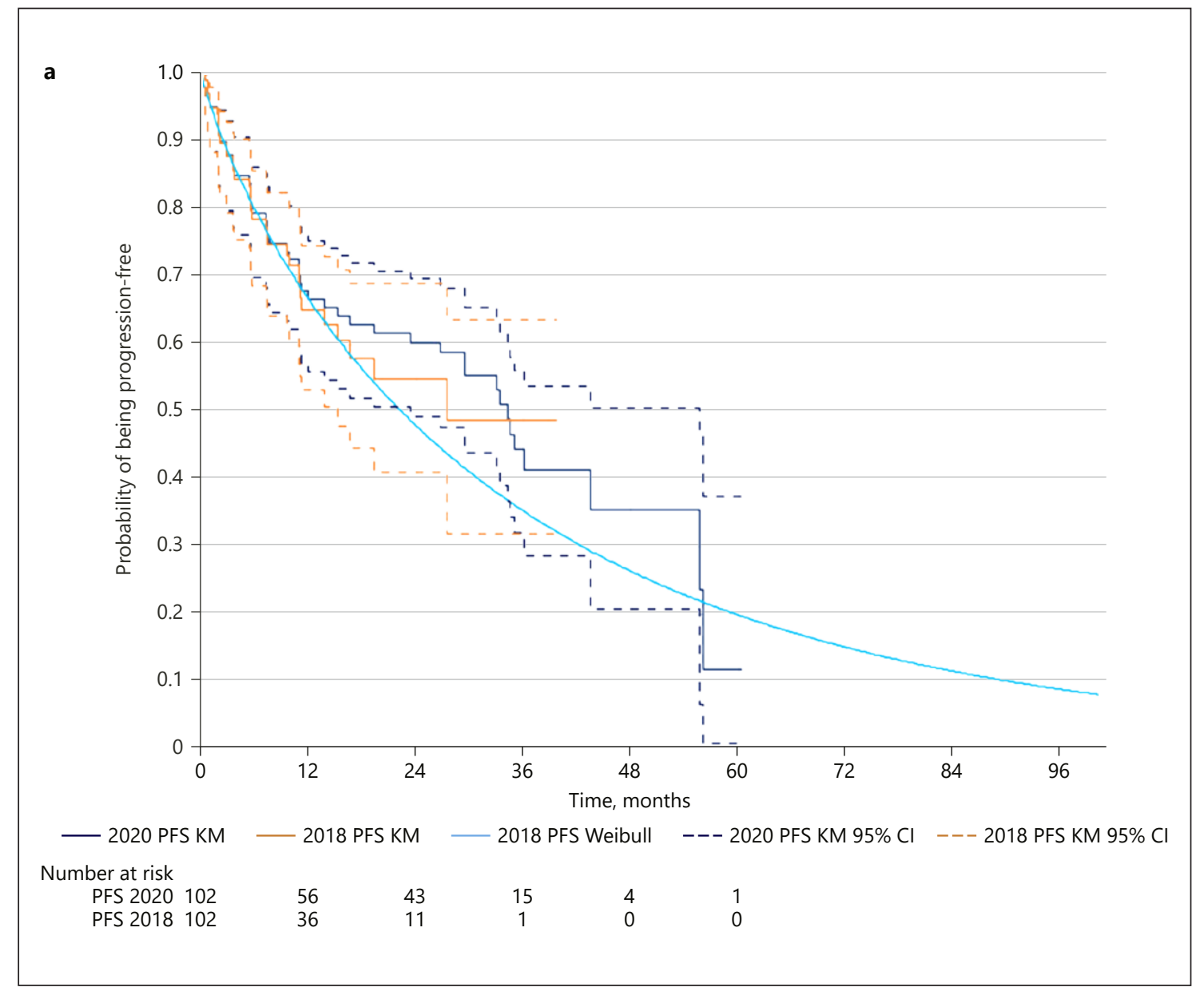

Fig. 1. a, b Comparison of 2018 and 2020 PFS and OS Kaplan-Meier data with 2018 PFS and OS Weibull predictions. OS, overall survival; PFS, progression-free survival; KM, Kaplan-Meier; CI, confidence interval.

(Figure continued on next page.)

Table 2. Patient characteristics that differ across 2018 and 2020 data cuts

\begin{tabular}{|c|c|c|}
\hline Characteristic & 2018 data cut $(N=102)$ & 2020 data cut $(N=102)$ \\
\hline Median duration of follow-up for OS, months & 15.61 & 36.70 \\
\hline \multicolumn{3}{|l|}{ Best overall response to larotrectinib, $n$ (\%) } \\
\hline Complete response & $15(14.71)$ & $20(19.61)$ \\
\hline Surgical complete response & $1(0.98)$ & $4(3.92)$ \\
\hline Partial response & $51(50.0)$ & $42(41.18)$ \\
\hline Stable disease & $12(11.76)$ & $12(11.76)$ \\
\hline Non-CR/Non-PD & $2(1.96)$ & $3(2.94)$ \\
\hline Progressive disease & $9(8.82)$ & $9(8.82)$ \\
\hline Not evaluable & $3(2.94)$ & $3(2.94)$ \\
\hline Missing & $9(8.82)$ & $9(8.82)$ \\
\hline
\end{tabular}

$\mathrm{CR}$, complete response; PD, progressive disease; OS, overall survival. * Best overall response determined by Independent Review Committee assessment. 


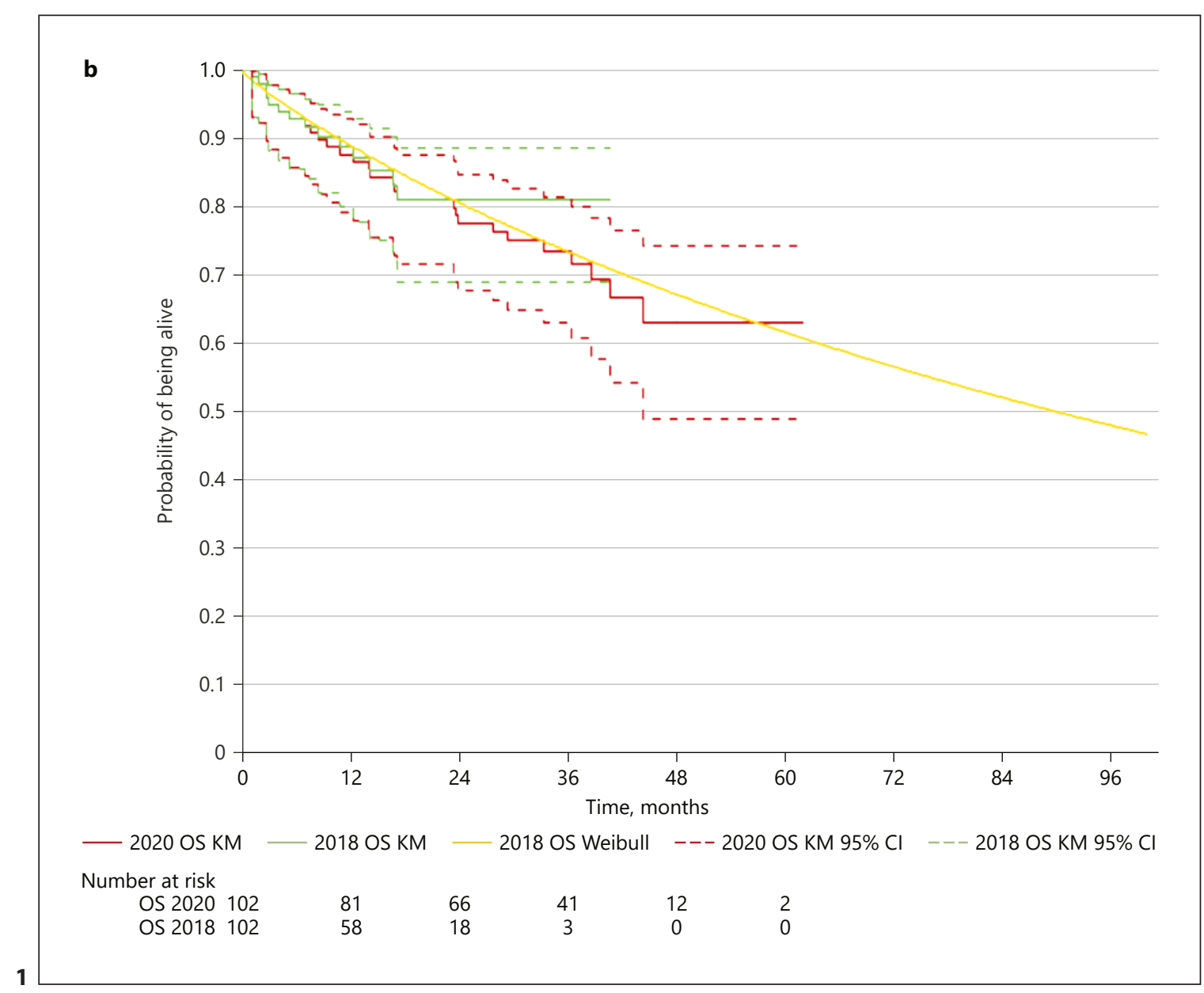

\section{Results}

\section{Patient Characteristics}

A total of 102 patients were included in the analysis, with a mean age of 37 years (standard deviation $=26.6$; shown in Table 1). Fifteen tumor types were represented, with the most common being soft tissue sarcoma (21\%), salivary gland carcinoma (17\%), infantile fibrosarcoma $(13 \%)$, and thyroid carcinoma (10\%).

When comparing patient characteristics across the 2018 and 2020 data cuts, the median follow-up time for OS increased by 21.1 months (shown in Table 2). The proportion of patients with complete response or surgical complete response was higher in the 2020 data cut (23.5\%) relative to the 2018 data cut (15.7\%).

\section{Progression-Free Survival}

Using the 2018 data cut, the predicted median PFS for larotrectinib was 22.4 months. In comparison, the median PFS for the $2020 \mathrm{KM}$ data was 33.4 months (shown in Fig. 1a). When comparing PFS from the survival prediction to observed $2020 \mathrm{KM}$ data, the 12 -month PFS rate was identical (66.6\%). The 36-month PFS rate prediction was an underestimate $(35.3 \%)$ relative to the $2020 \mathrm{KM}$ data $(44.4 \%)$, yet the prediction largely remained within the $95 \%$ confidence interval for the $2020 \mathrm{KM}$ data.

\section{Overall Survival}

Using the 2018 data cut, the median modeled OS for larotrectinib was 90.0 months (shown in Fig. 1b). The median OS was not yet reached in the $2020 \mathrm{KM}$ data. When comparing OS results from the prediction using the 2018 data to the observed $2020 \mathrm{KM}$ data, results were very similar, with 12 -month OS rate of $89.0 \%$ and $86.6 \%$, respectively, and 48 -month OS rate of $67.2 \%$ and $63.0 \%$, respectively. 


\section{Discussion/Conclusion}

We use 2 data cuts for the same patient dataset from basket trials for a tumor-agnostic therapy to evaluate the consistency of long-term survival predictions with observed longer follow-up data. This research suggests that modeling survival data may accurately predict longer term survival outcomes for tumor-agnostic therapies, particularly for OS. Additionally, it may be used to predict median survival when it has not yet been reached. Similar conclusions have been reached in previous studies when applying these methods to cancer immunotherapies $[10,11]$.

While PFS predictions largely remained within the $95 \%$ confidence intervals of the $2020 \mathrm{KM}$ data, the difference in the 36-month PFS rate was driven in part by response differences across tumor types and corresponding censoring patterns. For example, among the 9 primary central nervous system patients, 5 were censored in the 2018 data cut due to having achieved stable disease or partial response with no disease progression within the follow-up period. Those who were not censored had a median PFS of 3.7 months. In the 2020 dataset, only 2 central nervous system patients were censored with median PFS increasing to 6.3 months. Notably, these differences did not translate to inaccuracies in OS predictions. The survival prediction with the 2018 data was consistent with 2020 survival outcomes for OS.

Despite inherent uncertainty in long-term survival estimates, this analysis found that survival predictions from data with shorter follow-up were generally consistent with observed survival using data with longer follow-up among the same subset of patients. A limitation of this study is the low number at risk beyond 3-4 years, hindering the reliability of comparisons beyond this time point. Future research should explore the applications of these methods to other tumor-agnostic treatments to evaluate the extent to which this approach translates to other therapies and disease areas.

\section{Statement of Ethics}

No identifiable patient-level data were used in this analysis.

\section{Conflict of Interest Statement}

Dr. Briggs reports personal fees from Bayer during the conduct of the study, as well as personal fees from Amgen, Daiichi Sankyo, Merck, Pfizer, Roche, Rythmn, GSK, and Takeda outside the sub- mitted work. Ms. Rosettie reports funding from Bayer during the study period. Dr. Bokemeyer reports personal fees from Sanofi Aventis, Merck KgA, Bristol-Myers Squibb, Merck Sharp \& Dohme, Lilly Imclone, Bayer Healthcare, GSO Contract Research, AOK Rheinland-Hamburg, and Novartis outside the submitted work. Dr. Sullivan reports personal fees from Bayer outside the submitted work. Alexander Upton and Noman Paracha are employees of Bayer Healthcare Pharmaceuticals. No other disclosures were reported.

\section{Funding Sources}

This work was supported by Bayer Pharmaceuticals.

\section{Author Contributions}

All authors contributed to the design, planning, and conception of this study as well as to data interpretation and manuscript development. All authors reviewed the manuscript drafts and reviewed and approved the final version for submission.

\section{Data Availability Statement}

Availability of the data underlying this publication will be determined later according to Bayer's commitment to the EFPIA/ PhRMA "Principles for responsible clinical trial data sharing." This pertains to scope, time point and process of data access. As such, Bayer commits to sharing upon request from qualified scientific and medical researchers patient-level clinical trial data, study-level clinical trial data, and protocols from clinical trials in patients for medicines and indications approved in the United States (US) and European Union (EU) as necessary for conducting legitimate research. This applies to data on new medicines and indications that have been approved by the EU and US regulatory agencies on or after January 1, 2014. Interested researchers can use www.clinicalstudydatarequest.com to request access to anonymized patient-level data and supporting documents from clinical studies to conduct further research that can help advance medical science or improve patient care. Information on the Bayer criteria for listing studies and other relevant information is provided in the Study sponsors section of the portal. Data access will be granted to anonymized patient-level data, protocols and clinical study reports after approval by an independent scientific review panel. Bayer is not involved in the decisions made by the independent review panel. Bayer will take all necessary measures to ensure that patient privacy is safeguarded.
Briggs/Paracha/Rosettie/Upton/ Bokemeyer/Lassen/Sullivan 


\section{References}

1 Li IW, Krishnamurthy N, Wei G, Li G. Opportunities and challenges in developing tissue-agnostic anti-cancer drugs. J Cancer $\mathrm{Me}$ tastasis Treat. 2020;6:14.

2 Bayer. Highlights of Prescribing Information: VITRAKVI (larotrectinib) capsules, for oral use [updated March 2021. Available from: http: //labeling.bayerhealthcare.com/html/ products/pi/vitrakvi_PI.pdf.

3 European Medicine Agency. Vitrakvi. 2020. Available from: https://www.ema.europa.eu/ en/medicines/human/EPAR/vitrakvi.

4 Drilon A, Laetsch TW, Kummar S, DuBois SG, Lassen UN, Demetri GD, et al. Efficacy of larotrectinib in TRK fusion-positive cancers in adults and children. N Engl J Med. 2018; 378(8):731-9.
5 Hong DS, Bauer TM, Lee JJ, Dowlati A, Brose MS, Farago AF, et al. Larotrectinib in adult patients with solid tumours: a multi-centre, open-label, phase I dose-escalation study. Ann Oncol. 2019;30(2):325-31.

6 Laetsch TW, DuBois SG, Mascarenhas L, Turpin B, Federman N, Albert CM, et al. Larotrectinib for paediatric solid tumours harbouring NTRK gene fusions: phase 1 results from a multicentre, open-label, phase 1/2 study. Lancet Oncol. 2018;19(5):705-14.

7 Clark TG, Bradburn MJ, Love SB, Altman DG. Survival analysis part I: basic concepts and first analyses. Br J Cancer. 2003;89(2):232-8.

8 National Institute for Health and Care Excellence. Larotrectinib for treating NTRK fusion-positive solid tumours. Technology ap- praisal guidance [TA630]; 2020. Available from: https://www.nice.org.uk/guidance/ ta630.

9 Pocock SJ, Clayton TC, Altman DG. Survival plots of time-to-event outcomes in clinical trials: good practice and pitfalls. Lancet. 2002; 359(9318):1686-9.

10 Bullement A, Latimer NR, Bell Gorrod H. Survival extrapolation in cancer immunotherapy: a validation-based case study. Value Health. 2019;22(3):276-83.

11 Bullement A, Willis A, Amin A, Schlichting M, Hatswell AJ, Bharmal M. Evaluation of survival extrapolation in immuno-oncology using multiple pre-planned data cuts: learnings to aid in model selection. BMC Med Res Methodol. 2020;20(1):103. 